\title{
MICROGRAVITY ISOLATION SYSTEM DESIGN: A MODERN CONTROL SYNTHESIS FRAMEWORK
}

\author{
R. D. Hampton, Research Associate \\ C. R. Knospe, Assistant Professor \\ P. E. Allaire, Professor \\ Department of Mechanical, Aerospace, and Nuclear Engineering \\ University of Virginia \\ Charlottesville, VA 22903 \\ C. M. Grodsinsky \\ NASA Lewis Research Center \\ Cleveland, OH 44135
}

\begin{abstract}
Manned orbiters will require active vibration isolation for acceleration-sensitive microgravity science experiments. Since umbilicals are highly desirable or even indispensable for many experiments, and since their presence greatly affects the complexity of the isolation problem, they should be considered in control synthesis. In this paper a general framework is presented for applying extended $\mathrm{H}_{2}$ synthesis methods to the three-dimensional microgravity isolation problem. The methodology integrates control- and state frequency weighting and input- and output disturbance accommodation techniques into the basic $\mathrm{H}_{2}$ synthesis approach. The various system models needed for design and analysis are also presented. The paper concludes with a discussion of a general design philosophy for the microgravity vibration isolation problem.
\end{abstract}

\section{INTRODUCTION}

Although many scientists have planned or conducted materials processes and fluid physics science experiments designed for a weightless environment, the currently available facilities have proved far from ideal. Evacuated drop towers can provide only a few seconds of "weightlessness" at levels on the order of $10^{-6} \mathrm{~g}_{\mathrm{o}}$ (where $\mathrm{g}_{\mathrm{o}}$ is the gravitational acceleration at sea level). Aircraft flying low-gravity parabolic trajectories can extend the time to about 15-20 seconds, and sounding rockets can provide several minutes of a microgravity environment, but the goal of providing days, or even hours, for microgravity research has proved elusive. It was once hoped that the Space Shuttle could provide the desired environment, but such factors as manned activity, machine and structural vibrations, and thruster firings for orientation or reboost have resulted in acceleration levels generally unsatisfactory for the designed experiments. (Background excitations have been measured in the milli-g range.) In fact, the data from many experiments have been found unacceptable due to the poor acceleration environment.

Due to the low frequencies of greatest concern (below about $10 \mathrm{~Hz}$ ) the isolation problem is a largely unfamiliar one to vibration engineers; the requirement of a corner frequency of about $10^{-3} \mathrm{~Hz}$ is particularly vexing. Passive isolation is incapable of solving the isolation problem in this region, and even should a 
sufficiently soft spring be physically realizable, it could not isolate against direct disturbances to the payload. If the payload is tethered (e.g., for evacuation, power transmission, cooling, or material transport), a passive isolator cannot provide isolation below the corner frequency imposed by the umbilical stiffness.

An active isolator (such as a magnetic suspension system) that merely possesses a low positive stiffness fares no better in the presence of an umbilical, for the same reasons. And if the control system seeks to lower the corner frequency by adding negative stiffness, to counteract the umbilical's stiffness, the system will (at best) possess almost no stability robustness. In the face of the usual umbilical nonlinearities and uncertainties, this situation is clearly unacceptable. At very low frequencies the rattlespace constraints become limiting (refs. 1,2 ), so that any isolation system must have unit transmissibility in that region. In short, a microgravity isolator must be active, and it must be capable of dealing with the particular frequency-dependent complexities accompanying a tethered payload and a restrictive rattlespace.

The available acceleration data clearly point to a need for three-dimensional isolation (ref. 3 ). Classical control design methods are not well-suited for handling such problems; modern control methods provide a much more natural setting, opening up to the designer the power of the developing robust control synthesis and -analysis tools, along with a variety of well-tested and progressive computational software packages

The well-known $\mathrm{H}_{2}$ synthesis [i.e., LQR ("Linear Quadratic Regulator") or LQG("Linear Quadratic Gaussian")] methodology is one such modern control method. It can readily provide an optimal feedback controller for a linearized plant (i.e., payload plus umbilical) subject either to no exogenous input (LQR case) or to white noise disturbances only ( $\mathrm{LQG}$ case). An optimal control found by $\mathrm{H}_{2}$ synthesis minimizes a quadratic ("energy-type") cost function, or performance index. Such a performance index is quite appropriate for the microgravity isolation problem, since it allows penalizing both the control energy required for isolation and the vibrational energy of the payload. Unfortunately, however, the application of this synthesis method to practical problems has been plagued by robustness difficulties. Granted, the standard LQR solution provides excellent robustness guarantees for the single-input-single-output (SISO) problem (ref. 4, pp. 70-74) and also yields guarantees (though less useful) for the multiple-input-multipleoutput (MIMO) problem (ref. 5). But the addition of a state observer to the controller (as is usually necessary for practical problems) removes these robustness guarantees (ref. 6). This fundamental practical concern has led to a common skepticism regarding $\mathrm{H}_{2}$ synthesis.

There exist extensions to $\mathrm{H}_{2}$ synthesis, however, which can resolve the robustness issues. The disturbance-accommodation and frequency-weighting techniques contributed, respectively, by $\mathrm{C}$. D. Johnson (ref. 7, 1968; refs. 8 and 9, 1970; ref. 10, 1971) and N. K. Gupta (ref. 11, 1980) have proved to be highly useful in this regard. In fact, they provide the fundamental additional tools needed for solving practical controller design problems. These two extensions lead to augmented state equations which still allow for problem solution by the familiar $\mathrm{H}_{2}$ synthesis machinery. Recent investigations have examined the effect of the frequency-weighting extension on system robustness (refs. 12,13), and the dual relationship between frequency weighting and disturbance-accommodation (ref. 14). Additional extensions have also been proposed (ref. 15)

The utility of "extended $\mathrm{H}_{2}$ synthesis" for the tethered microgravity vibration isolation problem has been clearly demonstrated by recent studies (refs. 16, 17). Extended $\mathrm{H}_{2}$ synthesis has been used effectively to develop SISO and SIMO (single-input-multiple-output) controllers for a realistic onedimensional microgravity vibration isolation problem, using a "smart" form of acceleration feedback. The 
resulting closed-loop system exhibited excellent stability- and performance robustness guarantees, including a high degree of robustness to umbilical parametric uncertainty.

The present paper will give a general framework for controller design by the extended $\mathrm{H}_{2}$ synthesis method, for the microgravity vibration isolation problem. Following a summary of the basic $\mathrm{H}_{2}$ synthesis approach, the paper will describe how to incorporate the control- and state frequency weighting and inputand output disturbance accommodation extensions into the synthesis procedure. Control noise will also be included. General guidelines will be presented for effectively integrating these extensions into the design procedure.

The development below is specifically tailored to the microgravity isolation problem, but the mathematics are fully applicable to any problem that has the appropriate (very general) mathematical description. Four fundamental system models will be presented to aid the designer in visualizing the design effort. Only the synthesis procedure will be detailed here; the analysis techniques used for controller evaluation will be detailed in a later work.

\section{BASIC $\mathrm{H}_{2}$ SYNTHESIS REVIEW}

A generic microgravity vibration isolation system is depicted below in Fig. 1. A payload, such as a microgravity science experiment, is acted upon by actuators (typically non-contacting) that are commanded by a control system. This control system uses measurements, such as payload positions and accelerations, to develop the control signals, typically currents or voltages. The objective of $\mathrm{H}_{2}$ synthesis is to find a control signal that minimizes the weighted sum of the two-norm of the control energy and of the states, subject to the linearized system equations of motion. This control signal will be found to be dependent only on the past accumulative measurement information, for a system excited only by zero-mean white Gaussian noise.

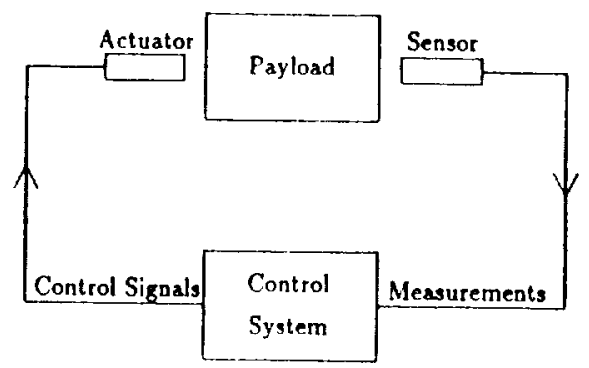

Figure 1. Vibration isolation system.

Specifically (and a bit more mathematically), to use $\mathrm{H}_{2}$ synthesis the system equations of motion must first be linearized and expressed in the following (standard) state space form:

$$
\underline{\dot{x}}=A \underline{x}+B \underline{u}+E_{s} \underline{w}_{s} \quad \underline{y}=\left(\underline{x}+D \underline{u}, \quad \underline{z}=\underline{y}+E_{n} \underline{w}_{n}\right.
$$

$\underline{x}$ is the state vector, $y$ is the output vector, $\underline{z}$ is the measurement vector, $\underline{w}$ is the control vector, $E_{s}$ and $F_{n}$ are selection matrices, and $\underline{w}_{S}$ and $\underline{w}_{n}$ are process- and sensor-noise vectors, respectively. For the microgravity vibration isolation problem, the process noise $\left(\underline{w}_{s}\right)$ models the disturbances acting on the payload, either directly (e.g., air currents, fluid flow, or experiment-mounted rotating machinery) or indirectly (i.e., through the umbilicals). The sensor noise $\left(\underline{w}_{n}\right)$ models the electrical or mechanical noise that contaminates the state measurements. In general, not all states will be measurable (i.e., rank $C^{*} \leq \operatorname{dim}$ $x)$. 
Let the initial conditions on the state vector be $\underline{x}(\theta)=\underline{x}_{0}$ (although these intitial conditions will not appear in the final control solution); let $\underline{x}_{0}, \underline{w}_{s}$, and $\underline{w}_{n}$ be independent and bounded (as is reasonable, since unbounded states and infinitely large noise are not physically possible); and let $\underline{x}_{0}$ be Gaussian, and $\underline{w}_{n}$ and W's, zero-mean white Gaussian, for technical reasons. The power of the process- and sensor-noise vectors can be expressed mathematically by

$$
\operatorname{cov}\left[\underline{w}_{s}(t), \underline{w}_{s}(\tau)\right]-V_{l} \delta(1-\tau) \text { and } \operatorname{cov}\left[\underline{w}_{n}(t), \underline{w}_{n}(\tau)\right]=V_{3} \delta(t-\tau)
$$

(ref. 18, p. 272). Assume that $\{A, B\}$ and $\left\{A, E_{s} V_{l}^{1 / 2}\right\}$ are stabilizable, where $V_{1}=V_{1}^{1 / 2} V_{1}^{1 / 2 *}$ (the asterisk here means "conjugate transpose"), and that $\{C, A\}$ is detectable (ref. 19, p. 226). These requirements mean, respectively, that a stabilizing controller exists, and that the available measurements are sufficient for its implementation. Let $V_{1}$ and $V_{3}$ be positive semidefinite (PSD) and positive definite (PD), respectively, for reasons of solution existence. That is, there need not be any process noise, but there must be at least some noise in all measurement channels (as there will be) if an optimal control solution is to exist.

The $\mathrm{H}_{2}$ synthesis design method uses a quadratic performance index,

$$
J=\lim _{T \rightarrow \infty} \frac{1}{2 T} \int_{0}^{T}\left\{\varepsilon\left\langle\left[\underline{x}^{T} \underline{u}^{T}\right]\left[\begin{array}{cc}
W_{1} & W_{2} \\
W_{2}^{T} & W_{3}
\end{array}\right]\left\{\begin{array}{l}
\underline{x} \\
\underline{u}
\end{array}\right\}\right)\right\} d t
$$

where $W_{1}, W_{2}$, and $W_{3}$ are weighting matrices. These weighting matrices, assigned by the designer, allow him to place a relative importance on the two-norm of each state (using $W_{1}$ ) and of the control (using $W_{3}$ ). $W$, allows him to assign cross weightings. (These cross-weightings are not generally used for the basic $\mathrm{H}_{2}$ synthesis problem, but they become important with some of the extensions.) $W$, is PSD and $W_{3}$ is PD (ref. 18; pp. 272, 276), again for reasons of solution existence. " $\rho "$ is the expected-value operator, needed since the system is excited stochastically by $\underline{w}_{s}$. The cost rate functional form for $J$ (with " $\lim _{T \rightarrow \infty} \frac{1}{2 T}$ " ) is used to allow for the white noise disturbance $\underline{w}_{s}$. Otherwise the performance index would be infinite.

Let an admissible control $\underline{u}(r)$ be one that depends only on the past accumulative observation data. That is, $\underline{v}(t)$ has the form

$$
\underline{u}(t)=\underline{u}[t, \underline{Z}(t)], \text { where } \underline{Z}(t)=\{\underline{z}(\tau), 0 \leq t \leq t\} .
$$

(For more general conditions on admissibility, see ref. 18, p. 272.) The objective is to find an admissible control function $\underline{u}^{*}(f)$ which minimizes the cost $J$ with respect to the set of admissible control functions u( $(f)$ subject to the dynamic constraint $(1 \mathrm{a}, \mathrm{b}, \mathrm{c})$. That is, the optimal control solution must exist and be realizable, must minimize the cost functional specified by the designer, and must take into consideration the system equations of motion

The solution is well-known, and is summarized as follows:

$$
\begin{aligned}
& \underline{u}^{*}(t)=-K \underline{\tilde{x}}(t) \\
& \text { where } \underline{\tilde{x}} \text { is an estimate of } \underline{x} \text { using a Luenberger observer } \\
& \quad \text { (ref. } 18, \mathrm{pp} .288-289) \text { having observer gain matrix } I . \\
& K=W_{3}^{-1}\left(B^{T} P+W_{2}^{T}\right) \\
& P \text { is the unique PD) solution } \\
& \quad \text { to the Algebraic Riccati Equation }(\mathrm{ARE}) \\
& P A+A^{T} P-\left(P B+W_{2}\right) W_{3}^{-1}\left(P B+W_{2}\right)^{T}+W_{1}=O, \\
& I=Q C^{T}\left(E_{n} V_{3} E_{n}^{T}\right)^{-1} \\
& Q \text { is the unique PD solution to the } \wedge \mathrm{RE} \\
& A Q+Q A^{T}-Q C^{T}\left(F_{n} V_{3} E_{n}^{T}\right)^{-1}\left(Q+E_{s} V, E_{s}^{T}=O,\right.
\end{aligned}
$$




\section{$P$ exists if $\{A, B\}$ is stabilizable and $\{C, A\}$ is detectable or if the system is asymptotically stable, and \\ $Q$ exists if $\left\{A, E_{s}, V_{1} / 2\right\}$ is stabilizable and $\{C, A\}$ is detectable or if the system is asymptotically stable.}

Note that this control signal is developed by simply applying constant (negative) feedback gains $K$ to estimates of the system states. These estimates are themselves optimal in that they are the "closest" to the actual states, in terms of the expected value of the rms of the estimate error. They are produced in the controller from the control signal and the measurement vector, using constant observer gains $L$. Fig. 2 below presents this standard optimal controller (e.g., cf. ref. 20, pp. 356, 366) in block-diagram form.

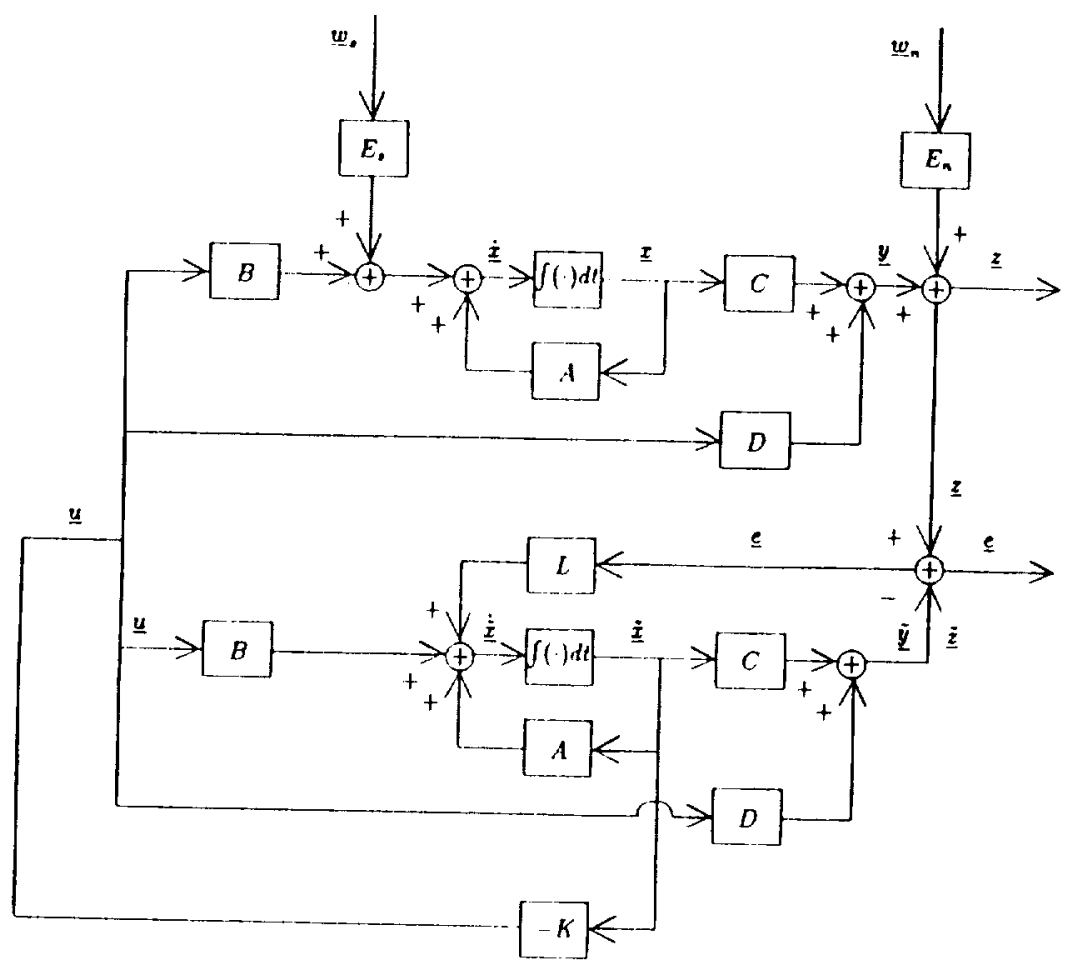

Figure 2. Block diagram of system with $\mathrm{H}_{2}$-optimal controller.

\section{EXTENSIONS $\mathrm{TO} \mathrm{H}_{2}$ SYNTHESIS}

\section{Frequency Weighting}

For the active microgravity vibration isolation problem, payload accelerations are of much greater concern at some frequencies than at others. Accelerations at higher frequencies can be handled passively, and very low-frequency accelerations correspond to such large displacements that they are essentially unisolable, due to practical rattlespace constraints. Rattlespace constraints also require that the relative displacements between space platform and payload be kept to a minimum at low frequencies. Control is needed at lower frequencies, where the plant is best-known and where the major isolation effort is desired At higher frequencies, however, excessive control can excite unmodeled higher modes of the plant Consequently it is desirable, through the performance index, to be able to penalize control strategies in a frequency-dependent fashion. This can be achieved by weighting the states $\underline{x}$ and the control $\underline{\not}$ in the cost 
rate functional so that the weightings are frequency-dependent. The latter, extended, $\mathrm{H}_{2}$ synthesis problem will be seen to have the same form as the former one. It will have the simple difference that the various system matrices now will be "augmented" to take into account the additional "pseudo" states required by the frequency-weighting extension.

Let $\underline{x}$ be considered to be the input to a filter $H_{(}(s)$ of which $\bar{x}$ is the output, and let $\mathscr{Y}(s)$ have a state-space representation defined by $\left\{A_{1}, B_{1}, C_{1}, D_{1}\right\}$.

That is, $\quad \mathscr{H}_{1}(s)-C_{1}\left(s \mathrm{~s}-A_{1}\right)^{-1} B_{1}+D_{1}$.

Then

$$
\underline{\dot{z}}_{1}=A_{1} \underline{z}_{1}+B_{1} \underline{x}, \quad \bar{x}=C_{1} \underline{z}_{1}+B_{1} \underline{x}
$$

expresses $\bar{x}$ in terms of $\underline{x}$, employing pseudostates $\underline{z}_{2}$. Similarly, if $\underline{u}$ is considered to be the input to a filter $Z_{3}(s)$ of which $\ddot{u}$ is the output, and if $\mathscr{H}_{3}(s)$ has a state-space representation defined by $\left\{A_{2}, B_{2}, C_{2}, D_{2}\right\}, \underline{u}$ can be expressed in terms of $\underline{\underline{u}}$, employing pseudostates $\underline{z} 2$ :

$$
\underline{\dot{z}}_{2}=A_{2} \underline{z}_{2}+B_{2} \underline{u}, \quad \underline{u}=C_{2} \underline{z}_{2}+D_{2} \underline{u}
$$

These frequency-weighred states $(\bar{x})$ and controls $(\bar{u})$ are now weighted (i.e., penalized) by constant weighting matrices $W_{1}$ and $W_{3}$. respectively. The resulting state equations and performance index are as follows:

$$
\begin{aligned}
& { }^{\prime} \underline{\dot{x}}={ }^{\prime} A^{\prime} \underline{x}+{ }^{\prime} B \underline{u}+I^{\prime} \underline{w}_{s}, \quad \underline{y}={ }^{\prime} C^{\prime} \underline{x}+D^{\prime} \underline{u}, \quad \underline{z}=\underline{y}+E_{n} \underline{w}_{n} \\
& J=\varepsilon\left\{\lim _{T \rightarrow \infty} \frac{1}{2 T} \int_{0}^{r}\left\langle\left[{ }^{\prime} \underline{x}^{T} \underline{u}^{T}\right]\left[\begin{array}{cc}
{ }^{\prime} W_{1} & ' W_{2} \\
{ }^{\prime} W_{2}^{T} & { }^{\prime} W_{3}
\end{array}\right]\left\{\begin{array}{l}
{ }^{\prime} \underline{x} \\
u
\end{array}\right\}\right) d t\right\}
\end{aligned}
$$

where ${ }^{\prime} \underline{x}=\left[\begin{array}{c}\underline{x} \\ \underline{z}_{1} \\ \underline{z}_{2}\end{array}\right], \quad{ }^{\prime} A=\left[\begin{array}{ccc}A & O & 0 \\ B_{1} & A_{1} & O \\ O & O & A_{2}\end{array}\right], \quad{ }^{\prime} B=\left[\begin{array}{c}B \\ O \\ B_{2}\end{array}\right], \quad{ }^{\prime} C=\left[\begin{array}{lll}C & O & O\end{array}\right]$

${ }^{\prime} E_{\mathrm{s}}=\left[\begin{array}{l}E_{\mathrm{s}} \\ \sigma \\ O\end{array}\right], \quad{ }^{\prime} W_{1}=\left[\begin{array}{ccc}D_{1}^{T} W_{1} D_{1} & D_{1}^{T} W_{1} C_{1} & O \\ C_{1}^{T} W_{1} D_{1} & C_{1}^{T} W_{1} C_{1} & O \\ O & O & C_{2}^{T} W_{3} C_{2}\end{array}\right], \quad{ }^{\prime} W_{2}=\left[\begin{array}{c}O \\ O \\ C_{2}^{T} W_{3} D_{2}\end{array}\right], \quad{ }^{\prime} W_{3}=\left[D_{2}^{T} W_{3} D_{2}\right] \quad(9 \mathrm{j}, \mathrm{k}, \mathrm{m}, \mathrm{n})$ The optimal control $\underline{u}(f)$ will now minimize the weighted sum of the two-norm of the frequency-weighted control energy and states.

\section{Input Disturbance Accommodation}

In the basic $\mathrm{H}_{2}$ problem it was assumed that the process noise $\underline{w}_{s}$ (i e., the disturbance acting on the payload, whether directly, or indirectly via the umbilicals) is zero-mean white Gaussian. This, of course, will not generally be the case; the process noise will have some (known or unknown) non-white power spectrum. Let the process noise be modeled as $\mathscr{L}_{s}$, where $\mathcal{L}_{s}$ is a stochastic disturbance with power spectral density $S_{f}(\omega)=S_{f}^{\prime \prime 2}(j \omega) S_{f}^{\prime \prime}(j \omega)$. Defining $H_{f}(j \omega)$ by $S^{\prime \prime 2}(j \omega) V_{f}^{\prime / 2}$, one can consider $f_{s}$ to be the 
density $S_{f}(\omega)=S_{f}^{\prime / 2}(j \omega) S_{f}^{\prime / 2}(j \omega)$. Defining $H_{f}(j \omega)$ by $S^{\prime \prime 2}(j \omega) V_{f}^{\prime \prime 2}$, one can consider $\mathcal{L}_{s}$ to be the output of a filter $H_{f}(s)$, excited by zero-mean white Gaussian noise $\underline{w}_{s}$ with power $V_{l}$ (i.e., $\left.\operatorname{cov}\left[\underline{w}_{s}(r), \underline{w}_{s}(\tau)\right]=V, \delta(t-\tau)\right)$.

In state-space form, $\quad \underline{\xi}_{1}=A_{s} \underline{\xi}_{1}+B_{s} \underline{w}_{s} \quad \underline{f} \underline{s}_{s}=C_{s} \underline{\xi}_{1}+D_{s} \underline{w}_{s}$

such that

$$
H_{r}(s)=C_{s}\left(s l-A_{s}\right)^{-1} B_{s}+D_{s}
$$

Incorporating these new pseudostates $\left(\xi_{l}\right)$ into the state equations and performance index yields the further augmented $\mathrm{H}_{2}$ synthesis problem given below:

$$
\begin{aligned}
& { }^{2} \underline{\dot{x}}={ }^{2} A^{2} \underline{x}+{ }^{2} B \underline{u}+{ }^{2} E_{\mathrm{s}} \underline{w}_{s}, \quad \underline{y}={ }^{2} C^{2} \underline{x}+D \underline{u}, \quad \underline{z}=\underline{y}+E_{n} \underline{w}_{n} \\
& { }^{2} J=\varepsilon\left\{\lim _{T \rightarrow \infty} \frac{l}{2 T} \int_{n}^{T}\left\langle\left[\begin{array}{ll}
{ }^{2} \underline{x}^{T} & \underline{u}^{T}
\end{array}\right]\left[\begin{array}{cc}
{ }^{2} W_{l} & { }^{2} W_{2} \\
{ }^{2} W_{2}^{T} & { }^{2} W_{3}
\end{array}\right]\left\{\begin{array}{l}
{ }^{2} x \\
\underline{u}
\end{array}\right\}\right) d t\right\}={ }^{\prime} J \\
& \text { where }{ }^{2} \underline{x}=\left\{\begin{array}{l}
{ }^{\prime} \underline{x} \\
\underline{\xi}_{1}
\end{array}\right\}, \quad{ }^{2} A=\left[\begin{array}{cccc}
A & O & O & E_{s} C_{s} \\
B_{1} & A_{1} & O & O \\
O & O & A_{2} & O \\
O & O & O & A_{s}
\end{array}\right], \quad{ }^{2} B=\left[\begin{array}{l}
B \\
O \\
B_{2} \\
O
\end{array}\right] \\
& { }^{2} C=\left[\begin{array}{llll}
C & O & O & O
\end{array}\right], \quad{ }^{2} E_{s}=\left[\begin{array}{c}
E_{s} D_{s} \\
O \\
O \\
B_{s}
\end{array}\right] \\
& { }^{2} W_{1}=\left[\begin{array}{cccc}
D_{1}^{T} W_{1} D_{1} & D_{1}^{T} W_{1} C_{l} & O & O \\
C_{1}^{T} W_{1} D_{1} & C_{1}^{T} W_{1} C_{1} & O & O \\
O & O & C_{2}^{T} W_{3} C_{2} & O \\
O & O & O & O
\end{array}\right],{ }^{2} W_{2}=\left[\begin{array}{c}
O \\
O \\
C_{2}^{T} W_{3} D_{2} \\
O
\end{array}\right] \\
& { }^{2} W_{3}=\left[I_{2}^{T} W_{3} D_{2}\right]
\end{aligned}
$$

The optimal-control solution to this problem will minimize the frequency-weighted cost functional as before, with the plant now considered to be subject to the specified colored noise disturbance.

In actual space applications the power spectrum of the process noise may not be known. Fortunately, orbiter spectral vibration information need not be available for disturbance accommodation to be used The disturbances can be assumed to have whatever form the designer finds useful. For example, if he desires the controller not to respond to process noise above some frequency range, he might choose to model $H_{f}(s)$ as a lowpass filter.

\section{Output Disturbance Accommodation}

The same procedure can be employed to incorporate colored sensor noise into the extended $\mathrm{H}_{2}$ synthesis problem. No sensor will have the white-noise contamination assumed by the basic $\mathrm{H}_{2}$ problem. And the designer may even find it useful to shape the sensor noise filter in some non-physical way. For example, if payload acceleration measurements are known to be more accurate in one frequency range, and relative position measurements in another, he might choose his sensor noise filters appropriately to 
"inform" the observer of these facts. The resulting observer would tend to rely more heavily on the more accurate measurement(s) in a particular frequency range, in its state-reconstruction process.

Let the sensor noise vector be $f_{n}$, with selection matrix $E_{n}$, where $f_{n}$ is a stochastically modeled disturbance with power spectral density $S_{n}(j \omega)=S_{n}^{\prime \prime 2}(j \omega) S_{n}^{\prime \prime 2}(j \omega)$. As with the input disturbance $f_{s^{r}} f_{n}$ can be considered to be the output of a filter $H_{n}(s)$ excited by zero-mean white Gaussian noise $\underline{w}_{n}$ with power $V_{3}\left(\right.$ i.e., $\left.\operatorname{cov}\left[\underline{w}_{n}(t), \underline{w}_{n}(\tau)\right]=V_{3} \delta(t-\tau)\right)$. In state-space form,

$$
\underline{\xi}_{2}=A_{n} \underline{\xi}_{2}+B_{n} \underline{w}_{n}, \quad \underline{f}_{n}=C_{n} \underline{\xi}_{2}+D_{n} \underline{w}_{n}, \text { such that } H_{n}(s)=C_{n}\left(s I-A_{n}\right)^{-1} B_{n}+D_{n}
$$

For the extended $\mathrm{H}_{2}$ synthesis problem with state- and control frequency weighting (pseudostates $\underline{z}_{1}$ and $\underline{z}_{2}$, respectively), and with input- and output disturbance accommodation (pseudostates $\xi_{l}$ and $\xi_{2}$, respectively), the augmented state equations and the performance index are as follows:

$$
\begin{aligned}
& { }^{4} \underline{\dot{x}}={ }^{4} A^{4} \underline{x}+{ }^{4} B \underline{u}+{ }^{4} E_{s}{ }^{4} \underline{w}, \quad \underline{z}={ }^{4}\left({ }^{4} \underline{x}+{ }^{4}\right) \underline{u}+{ }^{4} E_{n}{ }^{4} \underline{w} n \\
& J=\varepsilon\left\{\lim _{T \rightarrow \infty} \frac{I}{T} \int_{0}^{T}\left\langle\left[{ }^{4} \underline{x}^{T} \underline{u}^{T}\right]\left[\begin{array}{cc}
{ }^{4} W_{1} & { }^{4} W_{2} \\
{ }^{4} W_{2}^{T} & { }^{4} W_{3}
\end{array}\right]\left\{\begin{array}{c}
{ }^{4} \\
\underline{u}
\end{array}\right\}\right) d t\right\} \\
& \text { where }{ }^{4} \underline{x}=\left\{\begin{array}{l}
\underline{x} \\
\underline{z}_{1} \\
\underline{z}_{2} \\
\underline{\xi}_{1} \\
\underline{\xi}_{2}
\end{array}\right\}, \quad{ }^{A} A=\left[\begin{array}{ccccc}
A & O & O & E_{s} C_{s} & O \\
B_{1} & A_{1} & O & O & O \\
O & O & A_{2} & O & O \\
O & O & O & A_{s} & O \\
O & O & O & O & A_{n}
\end{array}\right], \quad{ }^{A} B=\left[\begin{array}{c}
B \\
O \\
B_{2} \\
O \\
O
\end{array}\right] \\
& { }^{4} C=\left[\begin{array}{lllll}
C & O & O & O & E_{n} C_{n}
\end{array}\right], \quad{ }^{4} D=D, \quad{ }^{4} E_{s}=\left[\begin{array}{ccc}
E_{s} D & O \\
O & O \\
O & O \\
B_{s} & O \\
O & B_{n}
\end{array}\right] \\
& { }^{4} \underline{w}_{s}=\left\{\begin{array}{l}
\underline{w}_{s} \\
\underline{w}_{n}
\end{array}\right\}, \quad{ }^{4} \underline{w}_{n}=\left\{\underline{w}_{n}\right\}, \quad{ }^{4} E_{n}=\left[E_{n} D_{n}\right] \\
& { }^{A} W_{1}=\left[\begin{array}{ccccc}
D_{1}^{T} W_{1} D_{1} & D_{1}^{T} W_{1} C_{1} & O & O & O \\
C_{1}^{T} W_{1} D_{1} & C_{1}^{T} W_{1} C_{1} & O & O & O \\
O & O & C_{2}^{T} W_{3} C_{2} & O & O \\
O & O & O & O & O \\
O & O & O & O & O
\end{array}\right],{ }^{4} W_{2}=\left[\begin{array}{c}
O \\
O \\
C_{2}^{T} W_{3} D_{2} \\
O \\
O
\end{array}\right] \\
& { }^{4} W_{3}=\left[I_{2}^{T} W_{3} D_{2}\right] \\
& { }^{4} V_{1}=\left[\begin{array}{ll}
V_{1} & 0 \\
O & V_{3}
\end{array}\right] \text { is the autocorrelation matrix for }{ }^{4} w_{s} \text {, } \\
& { }^{A} V_{2}=\left[\begin{array}{l}
V_{2} \\
V_{3}
\end{array}\right] \text { is the cross correlation matrix between }\left\{\begin{array}{l}
\underline{w}_{s} \\
\underline{w}_{n}
\end{array}\right\} \text { and } \underline{w}_{n} \text {, } \\
& \text { and } V_{3}=V_{3}^{r} \text { is the autocorrelation matrix for } \underline{w}_{n} \text {. }
\end{aligned}
$$




\section{Control Noise}

The active microgravity vibration isolation system must perform well even when the actual system dynamics are less than perfectly described by the system model. One way to improve the isolation system's robustness to parameter changes at the control inputs (i.e., to uncertainties in the actuator or plant model) is to add a process noise input $\left(\underline{w}_{c}\right)$ to the model's control signal (ref. $21, \mathrm{p}$. 1-48). Recall that the $\mathrm{H}_{2}$ synthesis machinery seeks to minimize the rms of the observation error. It does this by finding an observer gain matrix $L$, that will optimally trade off the measurement uncertainties against the plant model uncertainties, in the state reconstruction process. Control noise will reduce the observer's "confidence" in the plant model, so that the observer will "trust" its measurement data more and its plant model less. The resulting gain matrix $L$ will sacrifice a degree of observation quality for improved observer robustness to plant model inaccuracies. The controller gain matrix $K$ will be unaffected.

Under these circumstances the state equations of motion, unaugmented by frequency weighting or disturbance accommodation, become

$$
\underline{\dot{x}}=A \underline{x}+B\left(\underline{u}+\underline{w}_{c}\right)+E_{s} \underline{f}_{s}, \quad \underline{y}=C \underline{x}+D\left(\underline{u}+\underline{w}_{c}\right), \quad \underline{z}=\underline{y}+E_{n} \underline{f}_{n}
$$

where $f_{s}$ and $f_{n}$ can be represented by filters in state-space form with white-noise inputs, as noted

previously. Assume no cross-correlation between $\underline{\underline{w}}_{c}$ and $\underline{w}_{s}$, or between $\underline{w}_{c}$ and $\underline{w}_{n}$, and let

$$
\operatorname{cov}\left[\underline{w}_{c}(t), \underline{w}_{c}(\tau)\right]=V_{c} d(t-\tau) \text {. }
$$

Using now the pre-superscript designator " 5 " to indicate the appropriate state-space augmentation, the system equations change as follows:

$$
\begin{aligned}
& { }^{5} \underline{x}={ }^{4} \underline{x}, \\
& { }^{5} A={ }^{4} A,{ }^{5} B={ }^{4} B,{ }^{5} C={ }^{4} C,{ }^{5} D={ }^{4} D, \\
& { }^{5} W_{1}={ }^{4} W_{1},{ }^{5} W_{2}={ }^{4} W_{2},{ }^{5} W_{3}={ }^{4} W_{3} \\
& { }^{5} E_{s}=\left[\begin{array}{ccc}
B & E_{s} D_{s} & O \\
O & O & O \\
B_{2} & O & O \\
O & B_{s} & O \\
O & O & B_{n}
\end{array}\right],{ }^{5} \underline{w}_{s}=\left\{\begin{array}{l}
\underline{w}_{c} \\
\underline{w}_{s} \\
\underline{w}_{n}
\end{array}\right\},{ }^{5} \underline{w}_{n}=\left\{\begin{array}{l}
\underline{w}_{c} \\
\underline{w}_{n}
\end{array}\right\},{ }^{5} E_{n}=\left[\begin{array}{ll}
D & E_{n} D_{n}
\end{array}\right] \\
& { }^{s} V_{l}=\left[\begin{array}{lll}
V_{c} & O & O \\
O & V_{1} & O \\
O & O & V_{3}
\end{array}\right], \quad{ }^{5} V_{2}=\left[\begin{array}{ll}
V_{c} & O \\
O & V_{2} \\
O & V_{3}
\end{array}\right], \quad{ }^{5} V_{3}=\left[\begin{array}{ll}
V_{c} & O \\
O & V_{3}
\end{array}\right]
\end{aligned}
$$

The basic tools are now in place for practical microgravity vibration isolation system design, by extended $\mathrm{H}_{2}$ synthesis.

\section{SYSTEM MODELING}

The $\mathrm{H}_{2}$ synthesis problem is actually a two-fold design problem; the designer must determine a regulator gain matrix $K$, and also an observer gain matrix $L$, which together are used to comprise the optimal controller. The full augmented state vector must be used for the regulator sub-design problem, 
but pseudostates $\underline{z}_{1}$ and $\underline{z}_{2}$ (which occur due to frequency weighting) need not be reconstructed by the observer. They can simply be developed by passing the reconstructed state vector $\underline{x}$ and the control vector $\underline{1}$, respectively, through the appropriate frequency-weighting filters. Consequently the observer sub-design problem can (though need not) be one of reduced order. It is helpful, then, to have different mathematical models for conceptualizing these two sub-problems. These models will also differ, in general, from the basic plant model, which depicts the "actual" linearized plant (i.e., the system without the controller) in state space form. This model will not include the frequency-weighting and disturbance-accommodation augmentations of the former. In addition to these three conceptual models of the system, there is a fourth model, which more properly falls under the category of analysis but should be kept in mind during the synthesis procedure. This "nominal analysis model" depicts the linearized and unaugmented plant with the synthesized controller attached. It is used, with various modifications, to analyze closed-loop system performance.

\section{Basic Plant Model}

The basic plant model (shown schematically in Fig. 3) simply presents the linearized differential equations of motion in a state space form useful to the $\mathrm{H}_{2}$ synthesis machinery. Such a representation is given below.

$$
\underline{\dot{x}}=A \underline{x}+B \underline{u}+E_{s} \underline{f}, \quad \underline{y}=C \underline{x}+D \underline{u}, \quad \underline{z}=\underline{y}+E_{n} \underline{f}_{n}
$$

No performance index is needed at this stage, since it is the frequency-weighted states and control which will be weighted relative to each other for the actual controller synthesis. For the microgravity vibration isolation problem a useful choice for the state vector would include relative displacements, relative velocities, and accelerations. Weighting an acceleration more heavily in a frequency range would correspond roughly to a demand to increase the associated effective mass (or inertia) of the system . A similar correspondence can be drawn between relative-displacement weighting and the effective relative stiffness, and between relative-velocity weighting and the effective relative damping.

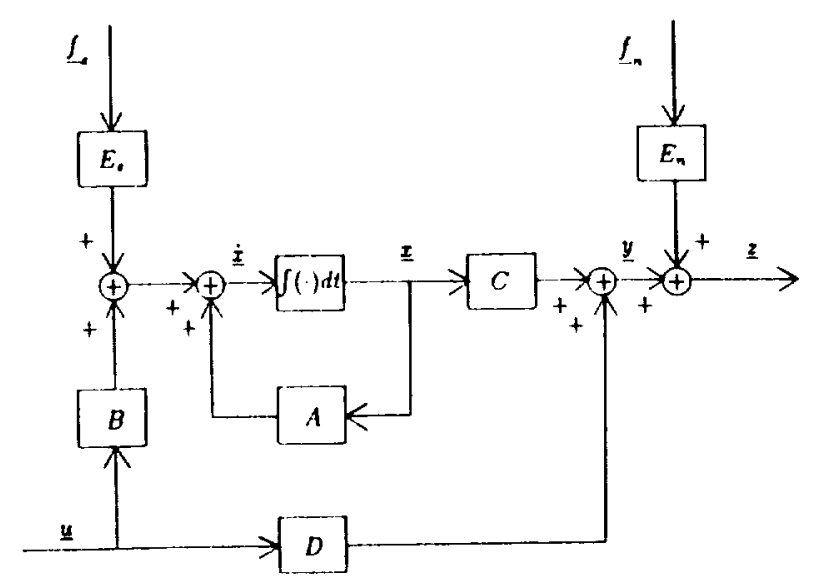

Figure 3. Basic plant model.

Regulator Synthesis Model

The regulator synthesis model adds frequency weighting and disturbance accommodation weighting filters to the basic plant model, and is the model actually used by the extended $\mathrm{H}_{2}$ synthesis machinery in 
designing the regulator. Fig. 4 portrays this model in block diagram form. Note that it has a similar form to the basic plant model, but that now augmented $A, B, C, E_{s_{1}} E_{n}, W_{1}, W_{2}$ and $W_{3}$ matrices are used, as indicated by the pre-superscripts. These matrices were defined previously ( $16 \mathrm{~b}$ through $16 \mathrm{~g}$ ). Also note that white noise vector $\underline{w}_{s}$ replaces $f_{s}$, since the process noise power spectral information is now contained in matrices ${ }^{5} A$ and ${ }^{5} E_{s}$.

The regulator synthesis model shows the system as viewed by the extended $\mathrm{H}_{2}$ machinery in determining the matrix ${ }^{5} P$. This matrix is the unique positive definite solution to the following $A$ RE,

$$
{ }^{5} P{ }^{5} A+{ }^{5} A{ }^{T} P-\left({ }^{5} P{ }^{5} B+{ }^{5} W_{2}\right){ }^{5} W_{3}^{-1}\left({ }^{5} P{ }^{5} B+{ }^{5} W_{2}\right)^{T}+{ }^{5} W_{1}=O
$$

$P$ is used to find the regulator feedback gains. This model is typically used only in determining the regulator gains $-{ }^{5} \mathrm{~K}$. It is not generally used for the design of observer gains $(\cdot)_{L}$. (although it could be), since that design problem can be reduced to one of lower order, as noted before.

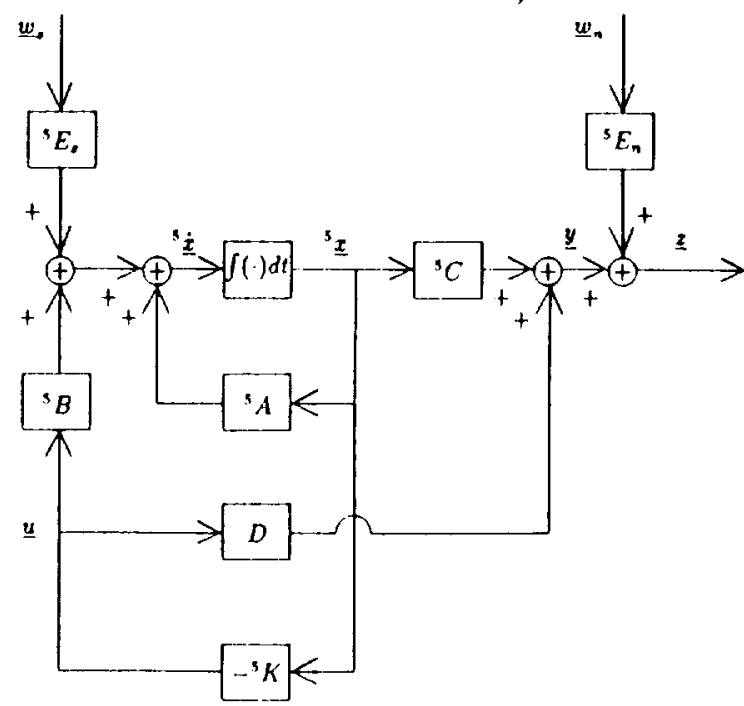

Figure 4. Regulator synthesis model.

Observer Synthesis Model

The observer uses the measurement vector $\underline{z}$ (e.g., measured relative positions and accelerations) and the control vector $\underline{u}$ as inputs to produce observations of the state vector. (In general an observer is needed to estimate the unmeasurable system states. Only rarely will all system states actually be available for measurement, and never will the disturbance-accommodation pseudostates actually be capable of measurement.) Kalman-Bucy filter design uses knowledge of process noise and sensor noise covariance matrices (and, if necessary, cross-correlation matrices) to produce optimal observer gains $(\cdot)_{l}$, in the sense of optimality previously discussed. If the complete augmented state vector ${ }^{5} \underline{x}$ is to be observed, then an appropriate observer synthesis model would be as depicted in Fig. 5. ${ }^{5} \mathrm{~T}$, would be found from the equation

$$
{ }^{S} L=\left({ }^{5} Q^{S} C^{T}+{ }^{5} E_{s}{ }^{S} V_{2}{ }^{5} E_{n}^{T}\right)\left({ }^{S} E_{n}{ }^{S} V_{3}{ }^{S} E_{n}^{T}\right)^{-1}
$$

where ${ }^{5} Q$ is the unique PD solution to the following $A R E$,

$$
{ }^{5} \tilde{A}^{5} Q+{ }^{5} Q^{5} \tilde{A}^{T}-{ }^{5} Q^{5} C^{5}\left({ }^{5} E_{n}{ }^{5} V_{3}{ }^{5} F_{n}^{T}\right)^{-1}{ }^{5} C^{5} Q+{ }^{5} E_{s}{ }^{5} \tilde{V}_{1}{ }^{5} E_{s}^{T}=O
$$

and where

$$
\begin{aligned}
& { }^{5} \tilde{A}={ }^{5} A-\left({ }^{5} E_{s}{ }^{5} V_{2}{ }^{5} E_{n}^{T}\right)\left({ }^{5} E_{n}{ }^{5} V_{3}{ }^{5} E_{n}^{T}\right)-1{ }^{5} \mathrm{C} \\
& { }^{s} \tilde{F}_{1}={ }^{s} V_{1}-{ }^{s} V_{2}{ }^{s} E_{n}^{T}\left({ }^{s} E_{n}{ }^{s} V_{3}{ }^{s} E_{n}^{T}\right)-1{ }^{s} E_{n}{ }^{s} V_{2}^{T}
\end{aligned}
$$


and ${ }^{5} V_{1},{ }^{s} V_{2},{ }^{5} V_{3}$, and ${ }^{5} E_{s}$ are as defined previously.

Equations $(19 \mathrm{~b}, \mathrm{c}, \mathrm{d})$ reduce to the form

$$
\begin{gathered}
{ }^{s} A{ }^{5} Q+{ }^{s} Q{ }^{s} A^{T}-\left({ }^{s} Q^{s} C^{T}+{ }^{s} E_{s}{ }^{s} V_{2}{ }^{5} E_{n}^{T}\right)\left({ }^{5} E_{n}{ }^{s} V_{3}{ }^{5} E_{n}^{T}\right)+\left({ }^{5} Q{ }^{5} C^{T}+{ }^{5} E_{s}{ }^{s} V_{2}^{T}{ }^{s} E_{n}^{T}\right) \\
+{ }^{s} E_{s}{ }^{s} V_{1}{ }^{s} E_{3}^{T}=O
\end{gathered}
$$

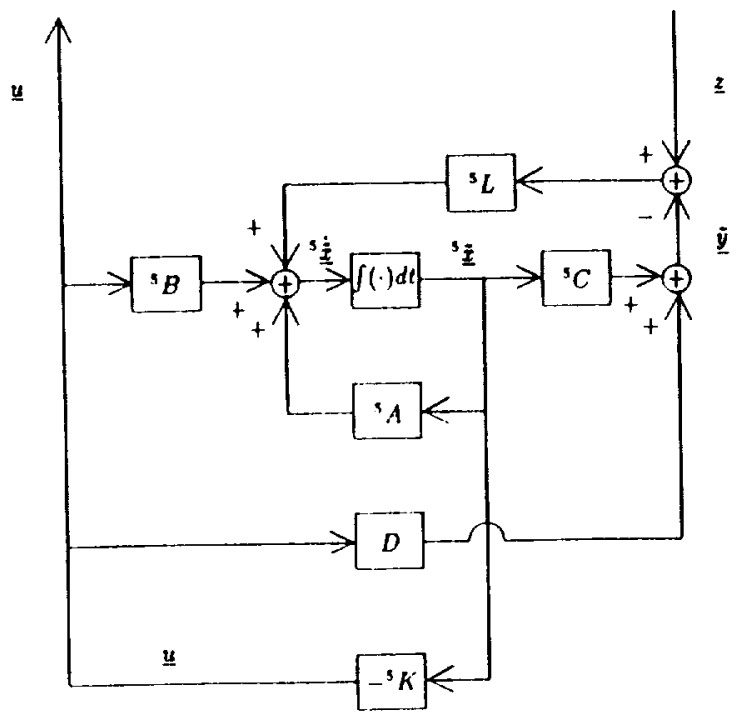

Figure 5. Observer synthesis model, observing all states and pseudostates

However, as noted previously, the observer does not need to reconstruct the frequency-weighting pseudostates $\underline{z}_{1}$ and $\underline{z}_{2}$. This fact will permit an observer of smaller dimension. In this case Fig. 6 will be an appropriate observer synthesis model, with pertinent matrices defined as follows. ${ }^{6} L$ would be found from the equation

$$
{ }^{6} L=\left({ }^{6} Q{ }^{6} C^{T}+{ }^{6} E_{s}{ }^{6} V_{2}{ }^{6} E_{n}^{T}\right)\left({ }^{6} E_{n}{ }^{6} V_{3}{ }^{6} E_{n}^{T}\right)^{-1}
$$

where ${ }^{\circ} Q$ is the unique PD solution to the following ARE,

$$
{ }^{6} \tilde{A}^{6} Q+{ }^{6} Q{ }^{6} \tilde{A}^{T}-{ }^{6} Q{ }^{6} C^{7}\left({ }^{6} E_{n}{ }^{6} V_{3}{ }^{6} E_{n}^{T}\right){ }^{-1}{ }^{6} C^{6} Q+{ }^{6} E_{s}{ }^{6} \tilde{V}_{1}{ }^{6} E_{s}{ }^{T}=O \text {, }
$$

and where

$$
\begin{aligned}
& { }^{6} \tilde{A}={ }^{6} A-\left({ }^{6} E_{s}{ }^{6} V_{2}{ }^{6} E_{n}^{T}\right)\left({ }^{6} E_{n}{ }^{6} V_{3}{ }^{6} E_{n}^{T}\right)^{-1}{ }^{6} C \\
& { }^{6} \tilde{V}_{1}={ }^{6} V_{1}-{ }^{6} V_{2}{ }^{6} E_{n}^{T}\left({ }^{6} E_{n}{ }^{6} V_{3}{ }^{6} E_{n}^{T}\right)-1{ }^{6} E_{n}{ }^{6} V_{2}^{T} \\
& { }^{6} V_{1}={ }^{5} V_{1},{ }^{6} V_{2}={ }^{6} V_{2}, \quad{ }^{6} V_{3}={ }^{5} V_{3} \\
& { }^{6} E_{s}=\left[\begin{array}{ccc}
B & E_{s} D_{s} & O \\
O & B_{s} & O \\
O & O & B_{n}
\end{array}\right],{ }^{6} A=\left[\begin{array}{ccc}
A & E_{s} C_{s} & O \\
O & A_{s} & O \\
O & O & A_{n}
\end{array}\right],{ }^{6} C=\left[\begin{array}{lll}
C & O & E_{n} C_{n}
\end{array}\right]
\end{aligned}
$$

Equations $(20 \mathrm{~b}, \mathrm{c}, \mathrm{d})$ reduce to the form

$$
{ }^{6} A^{6} Q+{ }^{6} Q^{6} A^{T}-\left({ }^{6} Q^{6} C^{T}+{ }^{6} E_{s}{ }^{6} V_{2}\right)^{6} V_{3}^{-1}\left({ }^{6} Q^{6} C^{T}+{ }^{6} E_{s}{ }^{6} V_{2}\right)^{T}+{ }^{6} E_{s}{ }^{6} V_{1}{ }^{6} E_{s}^{T}=O
$$




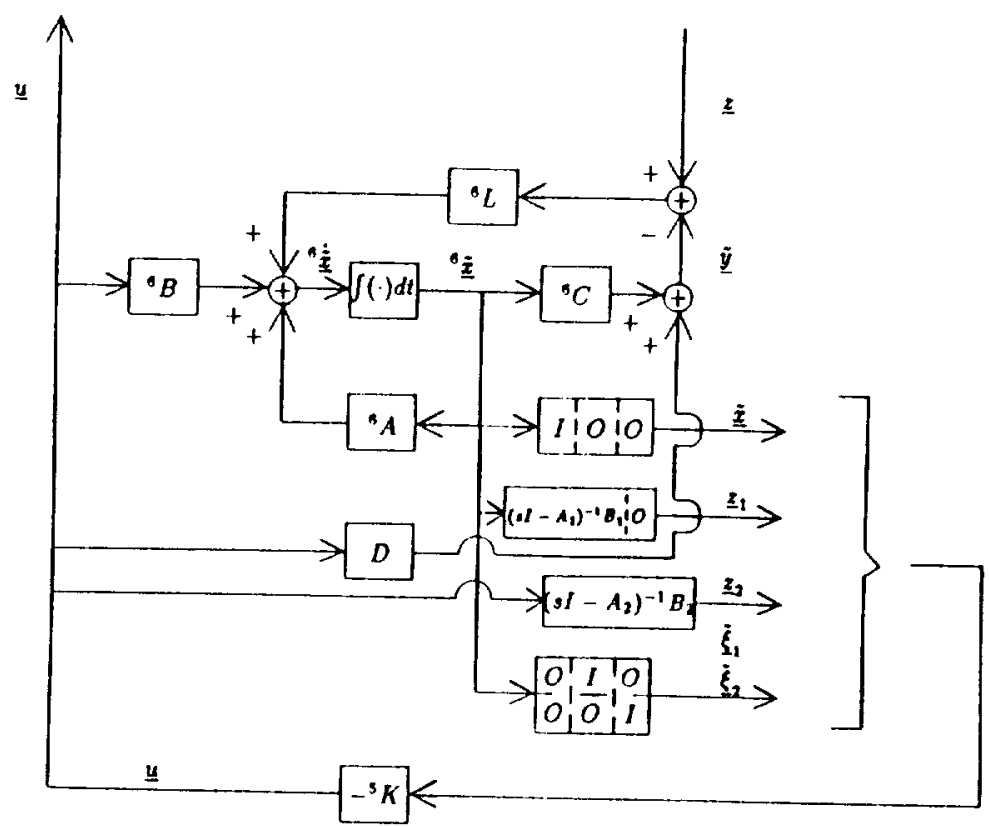

Figure 6. Reduced observer synthesis model.

\section{Nominal Analysis Model}

Once the active vibration-isolation controller has been designed, it can usually be reduced in size by modal truncation and/or balance-and-truncate ("Moore's method," ref. 22). Then the closed loop system can be evaluated, with the controller applied to the actual plant.

Letting the state space system $\left\{A_{F / B}, B_{F / B}, C_{F / B}, D_{F / B}\right\}$ represent the feedback controller, a nominal analysis model can be portrayed as in Fig. 7 . Checks on nominal stability can be made by simple eigenvalue

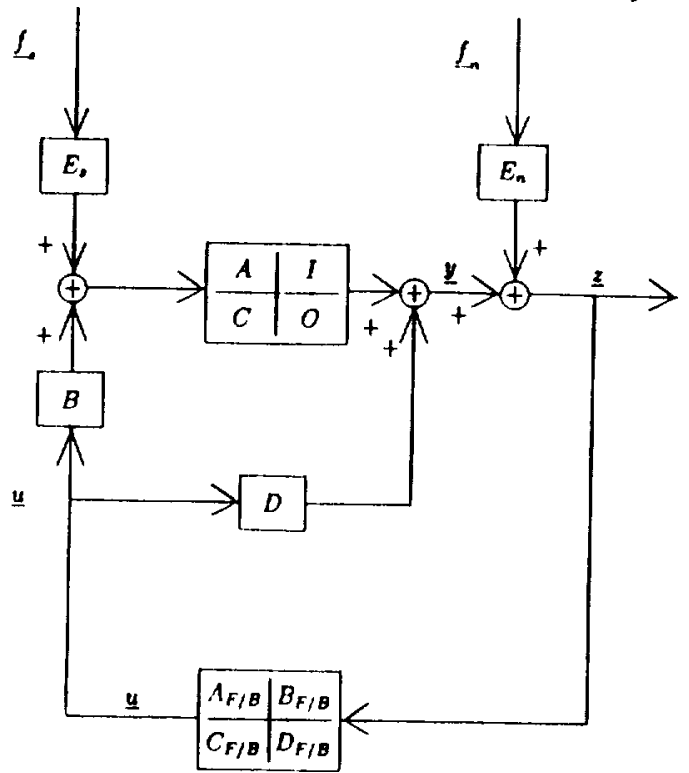

Figure 7. Nominal analysis model. 
checks of this closed-loop system. Nominal performance can also be conducted readily using this model. With the appropriate placement of complex $\Delta$-blocks to represent system uncertainties, one can also evaluate the system for robust stability and robust performance guarantees, using the powerful methods of mu-analysis (ref. 23).

\section{DESIGN PHILOSOPHY FOR THE MICROGRAVITY ISOLATION PROBLEM}

With the synthesis framework now in place, as presented above, the designer must choose a reasonable strategy in order to use his $\mathrm{H}_{2}$ synthesis tools with skill. He must determine what states to use, what frequency-weighting and disturbance-accommodation filters (if any) to employ, the relative weightings of the resultant frequency-weighted states and control, and the relative weightings of the various noise vectors in his system model. The designer must also decide which measurements to make, whether to use the full- or the reduced observer synthesis model, and whether to conduct the regulator-and observer-gain sub-problems in sequence or in combination.

One primary goal in controller design should be simplicity. The construction of the controller will be easiest, and its speed of operation fastest, if its complexity (i.e., the number of controller states) is kept to a minimum. To accomplish this aim, the authors recommend that the engineer seek to design the controller by starting with basic (unextended) $\mathrm{H}_{2}$ synthesis and adding complexity "one layer at a time." For example, he might first determine if basic LQG is adequate, and then add appropriate frequency weighting and disturbance accommodation step by step, evaluating after each addition whether or not the design is acceptable. If not, the next layer of complexity could be added based on the present design inadequacies. Once an adequate design has been found, it is recommended that the controller order (i.e., number of states) be reduced by using modal reduction and /or balance-and-truncate. This "step-up, step-down" philosophy should keep controller complexity to a minimum.

A second fundamental goal should be immitiveness. Unless the problem is posed in such a way as to employ the designer's intuition, he will find it very difficult, especially with a three-dimensional problem, to proceed with any degree of speed. The single most important step toward an intuitive problem is the proper choice of plant states. For the microgravity vibration isolation problem, the authors believe that a reasonably physical choice is payload relative position, payload relative velocity, and payload acceleration. $A$ heavier weighting on payload relative position (in the cost functional matrix $W_{1}$ ), for example, signals the $\mathrm{H}_{2}$ machinery to attempt to increase system stiffness. Similar analogues exist for the other two suggested states, as noted before. And at least two of these states are readily measurable for microgravity systems. Such state choices, then, allow the designer to assign his weightings with a degree of "physical feel," so that extended $\mathrm{H}_{2}$ synthesis becomes more of a craftsman's design tool, rather than a black box for use in a time-consuming trial-and-error approach.

The designer must also decide whether or not to conduct the regulator- and observer-gain design problems independently. The well-known "separation principle" guarantees that for a perfectly known system the regulator gains $K$ and the observer gains $L$ can be designed independently. One approach, then, would be first to design the regulator to meet the design goals, and then to design the observer to produce a state-vector estimate that is "accurate enough" over a "sufficient" bandwidth. The frequency-weighting and disturbance-accommodation extensions, however, affect the state observations in such a manner that "accurate enough" and "sufficient" are quite difficult terms to define. The closed-loop system must be analyzed as a whole for this purpose. The existence of an observer bandwidth can also be used to enhance overall system performance, so that a full-state-feedback system with inadequate performance can actually 
perform quite well when the observer is added. Since the system stability- and performance robustness must ultimately be evaluated for the total closed-loop system, it is recommended that the entire controller (i.e., the observer-plus-regulator) be designed as a unit, rather than in parts.

For the microgravity vibration isolation problem, studies to date indicate that $\mathrm{H}_{2}$ synthesis extensions are necessary, if one is to produce a practical control (ref. 16). This being so, there are certain frequency weightings that are very reasonable choices to use. At very low frequencies, indirect disturbances (i.e., orbiter positional deviations from a perfectly elliptical orbit), will be much larger than rattlespace constraints will allow. In the low-frequency region, then, the payload relative displacement should be weighted heavily, and the payload acceleration, lightly. These weighting choices could reasonably be expected to call for a controller producing unit transmissibility between orbiter and payload, at low frequencies. In the intermediate frequency range, where payload acceleration is of most concern, that state should be weighted heavily. At higher frequencies, where the plant model is not well known, high control weightings and low state weightings should be used to call for reduced control.

Certain disturbance-accommodation filters, as well, will be appropriate for the problem, while others will be inadvisable. From a physical perspective, a more massive experiment would be less susceptible either to direct or to indirect disturbances. One could expect, then, that an input disturbance filter which models a large direct disturbance would call for a controller tending to make the system seem more massive (electronically). On the other hand, an indirect disturbance alone (i.e., acting through the umbilical) could be attenuated effectively either by a greater system effective mass or by a reduced system effective stiffness. The latter means of disturbance attenuation is ineffective for direct disturbances. It also tends to reduce the stability robustness of the system. Hence, the designer should be wary of having too large an indirect disturbance model

Output disturbance accommodation and control noise should be included in the system model only if necessary. Research to date does not indicate that either is needed for microgravity vibration isolator design. Again, the goal is to achieve a satisfactory controller that is as simple as possible.

Observer design involves the numerical solution of an ARE. An ARE involving matrices of smaller dimension will be less susceptible to the numerical difficulties which sometimes attend such solution procedures. It is preferable, then, to use the reduced rather than the full observer synthesis model.

\section{CONCLUDING REMARKS}

Active vibration isolation of microgravity science experiments is a three-dimensional, MIMO design problem requiring sophisticated design- and analysis tools. Modern control methods provide the most natural setting for handling this problem, and with a suitable choice of states, modern-control design can be conducted in a relatively intuitive fashion. The $\mathrm{H}_{2}$ synthesis approach can be extended, using frequency weighting and disturbance accommodation techniques, to give the designer great flexibility in building a suitable controller. Implementation of these extensions involves a straightforward augmentation of various system matrices, so that the ARE-based solution methods of LQG synthesis can be readily applied. Extended $\mathrm{H}_{2}$ synthesis provides the necessary tools for the design of a robust isolation system. This paper has provided a general framework for using extended $\mathrm{H}_{2}$ synthesis to design the controller for such a system. 
In addition to the basic plant model, there are three complementary system models that are of use in conceptualizing the synthesis problem. Observer synthesis requires fewer pseudostates than regulator synthesis, so two respective system models are needed to reflect this difference. The controller model is developed by combining the observer and regulator models, followed by reduction of the controller dimensionality. Attachment of this controller to the basic plant model produces an analysis model that can be used, with mu-analysis methods, to evaluate the closed-loop system in terms of its stability-and performance robustness.

This paper has also suggested a general design philosophy for applying the extended $\mathrm{H}_{2}$ synthesis machinery to the particular design problem at hand. In addition to an overall design strategy, reasonable state choices were suggested, and basic practical guidelines were given for the effective use of frequencyweighting and disturbance-accommodation techniques.

\section{ACKNOWLEDGMENTS}

The authors would like to thank NASA Lewis Research Center and the Commonwealth of Virginia's Center for Innovative Technology for their funding of this work. This paper is dedicated in memory of Joseph Lubonski, who championed g-jitter awareness and microgravity research during the past halfdecade at NASA Lewis Research Center.

\section{REFERENCES}

1. Knospe, C.; and Allaire, P.: Limitations on Vibration Isolation for Microgravity Space Experiments. Jourmal of Spacecraft and Rockets, Vol. 27, No. 6, Nov.-Dec. 1990, pp. 642-646.

2. Knospe, C. R.; and Allaire, P. E.: Limits on the Isolation of Stochastic Vibration for Microgravity Space Experiments. Journal of Spacecraft and Rockets, Vol. 28, No. 2, March-April 1991, pp. 229237

3. Nelson, Emily S.: An Examination of Anticipated g-Jitter on Space Station and Its Effects on Materials Processes. NASA 1M-103775, April 1991.

4. Anderson, Brian D. O.; and Moore, John B.: Limear Optimal Conrrol Englewood Cliffs, New Jersey: Prentice Hall, Inc., 1971.

5. Safonov, M. D.; and Athans, M.: Gain and Phase Margins for Multiloop LQG Regulators. IEEE Transactions on Automatic Control, April 1977, pp. 173-179

6. Doyle, J. C.: Guaranteed Margins for LQG Regulators. IEEE Transactions on Auromatic Control, Vol AC-23, 1978, pp. 756-757.

7. Johnson, C. D.: Optimal Control of the Linear Regulator with Constant Disturbances. IEEE Transactions on Antomatic Control, Vol. AC-13, August 1968, pp. 416-421. 
8. Johnson, C. D.: Further Study of the Linear Regulator with Disturbances--The Case of Vector Disturbances Satisfying a Linear Differential Equation. IEEE Transactions on Automatic Control (Short Papers), Vol. AC-15, April 1970, pp. 222-228.

9. Johnson, C. D.: Further Comments on "Optimal Control of the Linear Regulator with Constant Disturbances." IEEE Transactions on Automatic Control, Vol. AC-15 August 1970, pp. 516-518

10. Johnson, C. D.: Accommodation of External Disturbances in Linear Regulator and Servomechanism Problems. IEEF Transactions on Automatic Control, Vol. AC-16, December 1971, pp. 635-644

11. Gupta, N. K.: Frequency-Shaped Cost Functionals: Extension of Linear-Quadratic-Gaussian Design Methods. AIAA Journal of Guidance and Control, November-December 1980, pp. 529-535.

12. Anderson, B. D. O.; and Mingori, D. L.: Use of Frequency Dependence in Linear Quadratic Control Problems to Frequency-Shape Robustness. Jommal of Gnidance, Control, and Dymamics, Vol. 8 , No. 3, May-June 1985, pp. 397-401.

13. Teo, C. L.; and Tomizuka, M.: Frequency-Shaped Cost Functionals: Output or Input Weighting. Proceedings of the 28rh Conference on Decision and Control, Tampa, Florida, December 1989, pp. 2389-2390.

14. Sievers, Lisa A.; and Von Flotow, Andreas H.: Comparison of Two LQG-Based Methods for Disturbance Rejection. Proceedings of the 28th (Conference on I)ecision and Control, Tampa, Florida, December 1989, pp. 483-485.

15. Hampton, R. D.; and Knospe, C. R.: Extended $\mathrm{H}_{2}$ Synthesis for Multiple-Degree-of- Freedom Controllers. Proceedings of the Imernational Symposium on Magnetic Suspension Technology, Hampton, Virginia, August 19-23, 1991, sponsored by NASA Langley Research Center.

16. Hampton, R. D:; Knospe, C. R.; and Grodsinsky, C. M.: Controller Design for Microgravity Vibration Isolation Systems (IAF-92-0969). World Space Congress 1992, Washington, D.C., August 28 - September 5, 1992.

17. Hampton, R. D.: Controller Design for Microgravity Vibration Isolation Systems. Ph.D. dissertation, University of Virginia, Charlottesville, Virginia, 1993.

18. Sage, A. P.; and White, C. C., III: Optimmm Systems Commol, 2nd ed. Englewood Cliffs, New Jersey: Prentice Hall, Inc., 1977.

19. Maciejowski, J. M.: Multi'ariable Feedhack Design. Wokingham, England: Addison-Wesley Publishing Company, Inc., 1989. 20. Chen, Chi-Tsong: Linear System Theory and Design. New York: Holt, Rinehart and Winston, Inc.,
1985.

21. Grace, A.; Laub, A. J.; Little, J. N.; and Thompson, C.: MATLAB User's Guide: Control System Toolbox. October 30, 1990, p. 1-48. 
22. Moore, B. C.: Principal Component Analysis in Linear Systems: Controllability, Observability, and Model Reduction. IEEE Transactions on Automatic Control, Vol. AC-26, 1981, pp. 17-32.

23. Dailey, R. Lane: Lecture Notes for the Workshop on $\mathrm{H}_{\infty}$ and $\mu$ Methods for Robust Control. Seattle, Washington: The Boeing Company. Presented at the 1990 American Control Conference, San Diego, California, May 21-22, 1990. 